\title{
A Node Scheduling Game based on Water Cycle Algorithm for Energy Efficient Wireless Sensor Networks
}

\author{
M. Karthiga \\ Dept. of CSE \\ PSG College of Technology \\ Coimbatore, India
}

\author{
R. Venkatesan, PhD \\ Prof.\& Head, Dept. of CSE \\ PSG College of Technology \\ Coimbatore, India
}

\begin{abstract}
Sensor Networks are expected to sustain for a long period of time with limited battery power. Among the many approaches used, Node Scheduling is effective in increasing the Network Lifetime. Existing node scheduling approaches perceives the happenings in the Sense region as a random phenomenon. But the proposed approach studies the behaviour of targets in its region and uses the acquired knowledge to predict future presence of the target in a locality. Simulation results prove the effectiveness of prediction. Once the locality is known in advance, the sensor motes participate in a game to achieve an energy efficient schedule for the nodes. A Pareto-optimal Node Schedule for the game is determined by a metaheuristic approach called the Water Cycle algorithm. Again the energy efficiency of the solution is proved by the simulation results.
\end{abstract}

\section{General Terms}

Energy Efficient Algorithm

\section{Keywords}

Wireless Sensor Network, Network Lifetime, Bayesian Probability, Game Theory, Pareto Optimality, Water Cycle Algorithm.

\section{INTRODUCTION}

A Wireless Sensor Network (WSN) is a network of computation constrained, memory constrained and energy constrained sensor nodes deployed in the Region of Interest (ROI) [1]. The energy scarcity has kindled the development of many energy efficient protocols for a WSN [2-7]. Sleep scheduling the sensor nodes is one such technique, where redundant sensor nodes among the densely deployed WSN are put in a low power deep sleep state. Many energy efficient sleep scheduling strategies have been developed recently.

In [8] Manju et al. have proposed a heuristic which iteratively builds sensor covers. These sensor covers have comparatively high energy and cover the maximum targets. A target is said to be covered, if and only if it is in the vicinity of at least one sensor cover. Sensor covers are built such that all the targets are covered. Only one sensor cover is active at any given time thus saving energy.

Jingwen et al. [9] have proposed a metaheuristic approach which solves the Node Scheduling as a multi-dimensional optimization problem. Maximum coverage set and minimum node set are two objectives solved. The cost function has accounted both and arrives at an optimal node set which saves energy. In [10] the authors have predicted the trajectory of a target based on its current direction and kinematics.

The major setbacks of existing Node Scheduling strategies are:
1. Only Initial Energy is considered for determining the Node Set. The Node Sets are developed centrally.

2. Majority of the Scheduling Strategies work with the assumption of fixed targets.

3. In case of moving targets, the algorithm is highly complex.

The proposed approach is a simple Node Scheduling strategy for tracking the moving targets. Animals and human beings stick to familiarity. Jakob et al. in his behavioral studies [11] describes that animals always show preference to familiar places and familiar paths. Unless its usual path is obstructed, an animal takes the same path almost daily. Similarly seismic occurrence in an earthquake prone zone triggers seismic activities in predictable areas. This has been proved by Andrew et al. [12]. Many natural phenomena have a pattern of occurrence and this has been proved time and again. The proposed approach is hence developed for such applications which exhibit a pattern of occurrence. The proposed Node Scheduling Game (NSG), learns the trajectory of the target, predicts its future trajectory by Bayesian Estimation. If the locality of the next event is predicted, the nodes elsewhere enter a deep sleep state, only entering the Active Sensor Group when there is a probability of event occurring in its region. Not all the sensor nodes in the Awake Region need to be Active for sensing. Only few sensor nodes (depending on the probability) are put in an Active state and they form the sensor cover. Since Lifetime of a Sensor Network is measured by the time of its First Node Death (FND), to prolong the lifetime nodes should die more or less around the same time. To achieve this, energy must be spent proportionately by the nodes. This is possible if the sensor cover is chosen every time based on the nodes' residual energy. To choose the sensor cover, a metaheuristic approach called the Water Cycle Algorithm has been used (WCA). Here, it is assumed that the WSN has been clustered with any one of the state of art clustering algorithms.

The remaining of this paper is organized as follows. Section 2 describes the Bayesian Estimation; Section 3 introduces Game Theory for Node Scheduling and the concept of Pareto Optimality. Section 4 elaborates the WCA. Section 5 analyses the results and Section 6 concludes the paper.

\section{BAYESIAN ESTIMATION}

Bayesian Estimation begins with knowing the Initial or Prior Probability of an event $\mathrm{P}(\theta)$. The observations from history determine the Conditional Probability $\mathrm{P}(\theta / \mathrm{D})$.

The Posterior Probability of an event is then given by Baye's theorem (Eq. 1). 


$$
P\left(\frac{D}{\theta}\right)=\frac{P(D)}{P(\theta)} * P\left(\frac{\theta}{D}\right)
$$

In target tracking application, the Prior Probability of an event is the Probability (Prob.) of sensing a target at a cluster. The target's trajectory is then observed and recorded. When the trajectory has been partially studied, related events are spotted from the recorded data i.e. $\mathrm{P}\left(\mathrm{C}_{1} / \mathrm{C}_{2}\right)$. This is the probability of a target being spotted at Cluster $\mathrm{C}_{1}$ given it is spotted by sensors at Cluster $\mathrm{C}_{2}$. When the learning becomes more mature, the accuracy of conditional probability increases. Now at some point in future, when an event is spotted by Cluster $\mathrm{C}_{1}$, combining the Initial probability $\mathrm{P}\left(\mathrm{C}_{1}\right), \mathrm{P}(\mathrm{C} 2)$ and the Conditional Probability $\mathrm{P}\left(\mathrm{C}_{1} / \mathrm{C}_{2}\right)$ the probability of an event occurring in Cluster $\mathrm{C}_{2}$ right after event spotted at Cluster $\mathrm{C}_{1}\left(\mathrm{P}\left(\mathrm{C}_{2} / \mathrm{C}_{1}\right)\right)$ is determined by Baye's Theorem. Similar to $\mathrm{C}_{2}$, all the remaining neighboring Clusters of $\mathrm{C}_{1}$ thus determine the probability of an event occurring in its range. Thus all the clusters likely to be impacted are alerted and only those impacted clusters stay awake for sensing. Hence by Bayesian estimation, only a chain of necessary clusters along the target's trajectory are alerted for sensing.

\section{NODE SCHEDULING GAME}

The Lifetime of a WSN is defined by its time of First Node Death (FND). Hence as described earlier, to prolong the Network Lifetime, the nodes should run out of energy more or less around the same time. This is achieved by bringing a balance in the depletion of energy among the sensor nodes. The sensors in a cluster vary from each other in terms of their Residual Energy. When the prob. of event occurring near a cluster is high, the Cluster is an Awake Region and the sensor nodes of the impacted cluster decide on the number of nodes to remain in an Active State. This is calculated by Eq. 2 .

$$
A=p * N
$$

In Eq. 2 ' $\mathrm{N}$ ' is the number of cluster members and 'p' is the probability of occurrence of an event in the cluster. To determine the ' $\mathrm{A}$ ' nodes that remain active and ' $\mathrm{N}-\mathrm{A}$ ' nodes that go to a low power state, the nodes start a Node Scheduling Game.

\subsection{Defining the Node Scheduling Game}

The Node Scheduling Game (NSG) for Sleep Scheduling is defined as follows.

Players: All the nodes of the impacted cluster participate in the game. The players are $\{\mathrm{CM} 1, \mathrm{CM} 2 \ldots, \mathrm{CMN}\}$.

Strategy: Each node has two strategies available to it. It is represented by $S=\{\mathrm{W}, \underline{\mathrm{W}}\}$. It can either WakeUp 'W' and remain in Sense State or does not Wakeup 'W'. Since a node $\mathrm{N}_{\mathrm{i}}$ can play either of the strategies with a positive probability, this is a mixed strategy game.

Payoff: It is the incentive each player receives for each of its strategies.

\subsection{Pareto-optimal Solutions}

In some games, few strategies (actions) are dominant over others. Elaborately, if a player has two strategies $S$ and $\mathrm{S}^{*}$, it can be said that $S$ strictly dominates $S^{*}$, when it is the case that, for all other strategy profiles (all possible combination of actions other players could take) the utility of player ' $\mathrm{i}$ ' is strictly higher when he chooses strategy $\mathrm{S}$ over $\mathrm{S}^{*}$. $\mathrm{S}$ is said to have a weakly dominance over $\mathrm{S}^{*}$, when its utility for playing $\mathrm{S}$ is at least as high as choosing $\mathrm{S}^{*}$, for all the strategy profiles chosen by other players.
Generally Nash Equilibrium provides the solution concept of a game. Nash Equilibrium is a stable state achieved by the players, where each player plays his/her best response, knowing the behavior of other players. Instead of players choosing their Best Response, taking a perspective from outside, there must be an outcome which is suited for all the players and is a stable solution. This stable solution which suits every player can be given by the concept of Pareto Optimality.

When outcome $\mathrm{O}$ is preferred over $\mathrm{O}^{\prime}$ for everybody and is strictly preferred over $\mathrm{O}$ ' for at least one player, then ' $\mathrm{O}$ ' Pareto-dominates $\mathrm{O}^{\prime}$. Hence an outcome $\mathrm{O}$ is said to be a Pareto - optimal solution if it isn't Pareto dominated by any other outcome. Intuitively it can be observed that there can be more than one Pareto-optimal outcome and in addition there has to be at least one Pareto-optimal outcome for a game. The set of Pareto-optimal outcomes form the Pareto-optimal Archives. The Water Cycle Algorithm determines the Paretooptimal Archive for the Node Scheduling Game. One solution from the Archive is chosen as the Node Scheduling Set for the impacted Cluster.

\section{WATER CYCLE ALGORITHM 4.1 Hydrological Cycle}

Water Cycle Algorithm (WCA) developed by Eskandar et. al [13] is a recently developed metaheuristic approach based on the observation of the water cycle process in nature. The WCA imitates the behavior of streams and rivers that flow downward to the sea. The Water Cycle Process in nature starts with raining. Rain drops join together to form streams and two or more streams join together and moves downhill to form bigger streams. These streams either join the rivers or enter the sea. Additionally, when glaciers and snow melt, the water enters the underground stream (aquifers) and flows downhill. The underground stream discharges to a lake or a river which flows to the sea eventually. Finally the water from the sea evaporates and along with water vapor from plants (as result of photosynthesis) forms the clouds. As a result of condensation, water from the clouds reaches the earth in the form of rain. The WCA mimics the behavior of this process (Fig. 1) in nature for solving optimization problems.

\subsection{Initial Population}

WCA like the Genetic Algorithm and Particle Swarm Optimization Algorithm is a population based Metaheuristic. The initial population in WCA is a collection of RainDrops. Each RainDrop (Eq. 3) represents a possible solution.

$$
\text { RainDrop }=\left[\mathrm{X}_{1}, \mathrm{X}_{2} \ldots \mathrm{X}_{\mathrm{N}}\right]
$$

If the optimization problem is $\mathrm{N}$ dimensional, each raindrop is a $1 \mathrm{XN}$ dimensional array (Eq. 3). 


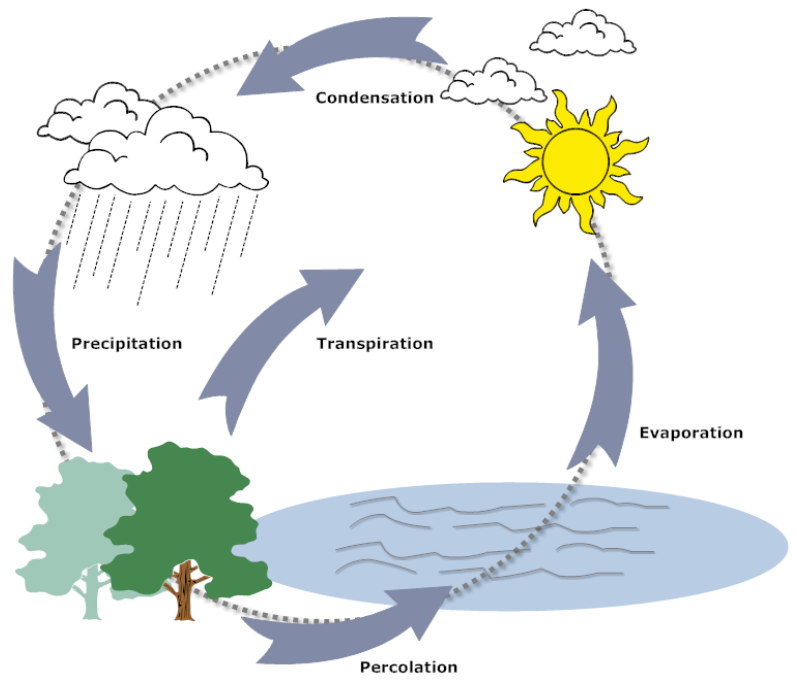

Fig: 1Water Cycle Process

The initial population (Eq. 4) is a randomly generated matrix of dimension pop_size $\mathrm{X} \mathrm{N}$, where pop_size is the size of initial population.

Initial Population $=\left[\begin{array}{ccc}X_{1}^{1} & \cdots & X_{N}^{1} \\ \vdots & \ddots & \vdots \\ X_{1}^{\text {pop_size }} & \cdots & X_{N}^{\text {pop_size }}\end{array}\right]$

Each design variable $X_{1}, X_{2} \ldots, X_{n}$ can be either a real valued number or a predefined set depending on the optimization problem to be solved. The aim of the Node Scheduling Game is to determine a highly energy efficient Node Set which also provides maximum coverage. Node scheduling game is a single dimensional optimization problem which strives to optimize the energy efficiency inside a cluster. Since NSG is a discrete optimization problem, the design variable $(\mathrm{X})$ is a randomly generated Node Set. Initial population is a matrix of raindrops with a single design variable namely the NodeSet which the forms the Sensor Cover. From Eq. 2 the number of nodes 'A' that remain active is determined. Each RainDrop hasa set of 'A' nodes chosen randomly among the ' $\mathrm{N}$ ' nodes.

\subsection{Cost Function}

The Cost function of each Raindrop is calculated by the following equation.

$$
\text { Cost }_{i}=f\left(X_{1}^{i}, X_{2}^{i}, \ldots, X_{N}^{\text {Pop_size }}\right)
$$

NSG being a single dimensional optimization problem designed to solve the energy efficiency problem, the cost function is the negative summation of Residual Energy of each node in the Node Set of the RainDrop (Eq. 6).

$$
\text { Cost }_{i}=-\sum_{j=1 \text { to } A} \text { Residual Energy }
$$

The aim of WCA is to minimize the cost of the solution.

\subsection{Streams and Rivers}

When 'Pop_size' raindrops are created, the raindrop with the minimum Cost $t_{i}$ becomes the Sea. Next best RainDrops in $\mathrm{N}_{\mathrm{sr}}$ become the rivers. The remaining RainDrops are the streams which flow directly to the sea or join the rivers. $\mathrm{N}_{\mathrm{sr}}$ is the summation of a sea and rivers.

$$
\begin{gathered}
\mathrm{N}_{\mathrm{sr}}=\text { Number of Rivers }+1 \text { (sea) } \\
\mathrm{N}_{\text {RainDrops }}=\text { Pop_size }-\mathrm{N}_{\mathrm{sr}}
\end{gathered}
$$

RainDrops are assigned to rivers and the sea. The number of raindrops $\left(\mathrm{NS}_{\mathrm{n}}\right)$ assigned to rivers and sea depends on the intensity of the flow which is given by Eq. 9 .

$$
N S_{n}=\operatorname{round}\left\{\mid \begin{array}{c}
\operatorname{Cost}_{n} / \sum_{i=1}^{N_{s r}} \operatorname{Cost}_{i} \mid * N_{\text {RainDropos }} \\
n=1,2, \ldots \ldots, N_{s r}
\end{array}\right\} \text {, }
$$

The Fig 2 represents the flow of streams to Sea and river. As described earlier, the raindrops join to form streams which join the rivers and everything finally ends up in the sea (the highest optimal point so far).

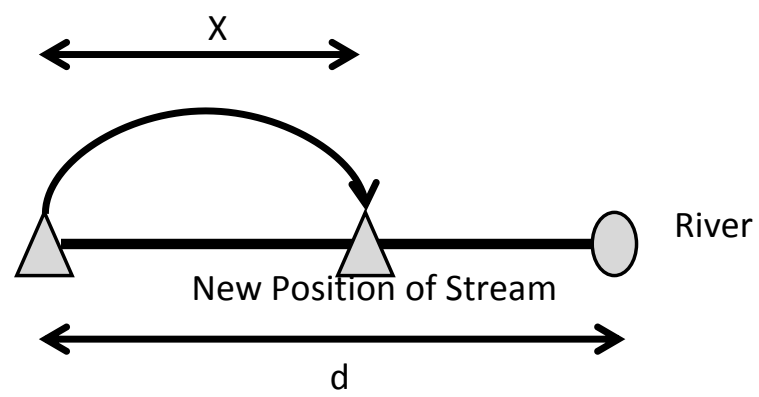

Figure 2 Flow of Stream towards River

As seen in the Fig. 2, the Streams join the Rivers or the Sea and the Rivers join the Sea along the connecting path (of distance d) existing between them. The stream joins the river by travelling a randomly chosen distance $\mathrm{X}$ calculated by Eq. 10. In Eq. 10, fixing a value greater than 1 for ' $C$ ' enables the streams to follow in varied paths towards the river. The same is extended for the rivers joining the sea

$$
\mathrm{X} \in(0, \mathrm{C} x \mathrm{~d}), \mathrm{C}>1
$$

The New positions of the Stream and River are calculated by Eq. 11 and Eq. 12.

$X_{\text {Stream }}^{i+1}=X_{\text {Stream }}^{i}+\operatorname{rand} X C X\left(X_{\text {River }}^{i}-X_{\text {Stream }}^{i}\right)$
$X_{\text {River }}^{i+1}=X_{\text {River }}^{i}+\operatorname{rand} X C X\left(X_{\text {Sea }}^{i}-X_{\text {River }}^{i}\right)$

If the new positions are better (based on cost function of the new streams and the new Rivers) than the Old Rivers and Sea their positions are interchanged (Fig. 3)

\subsection{Evaporation Condition}

Another important aspect of the WaterCycle process is evaporation. In nature water evaporates from Rivers/Lakes/Sea and also plants release water due to transpiration during photosynthesis. The evaporated water form clouds which condenses and the water reaches back as rain. 


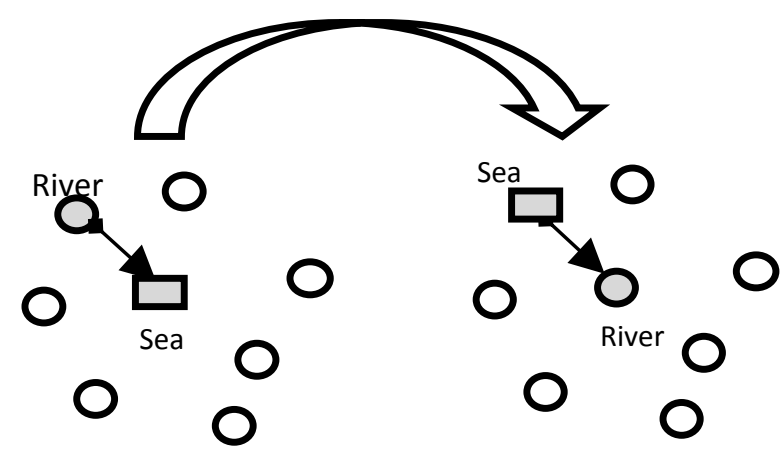

Fig. 3 Exchange of Positions of Sea and River

This is incorporated in the WCA to prevent the quick convergence of the algorithm and ending up in local optimum. When the algorithm satisfies the following condition, evaporation begins.

If $\left|X_{\text {sea }}^{i}-X_{\text {River }}^{i}\right|<d_{\max } \quad \mathrm{i}=1,2,3, \ldots, \mathrm{N}_{\mathrm{sr}}-1$.

Evaporation and Raining

End

If the distance between the Sea and a River is less than $d_{\max }$, evaporation condition is satisfied and precipitation starts. Lower value of $\mathrm{d}_{\max }$, increases the search intensity near the sea resulting in a highly optimal solution. The value of $d_{\max }$ iteratively decreases for each round.

$$
d_{\text {max }}^{i+1}=d_{\text {max }}^{i}-\frac{d_{\max }^{i}}{\max \text { iteration }}
$$

The precipitation begins with introducing new solutions as Raindrops which form the streams.

$$
X_{\text {new stream }}=L B+\operatorname{rand} X(U B-L B)
$$

In Node Scheduling Game the Lower Bound (LB) and Upper Bound (UB) constraints restricts the Raindrops to have sensor nodes which are members of the current cluster. The new Raindrops form the streams which join the Rivers or join the sea directly. The WCA is run till maximum number of iterations has been reached. At the end of each iteration, the Sea becomes a member of the Pareto Optimal Archive. At the end of each iteration, the non-dominated Pareto optimal solutions are retained and the dominated solutions are discarded. Table 1 describes the flow of WCA algorithm for determining the Pareto optimal Node Schedule. Column 2 shows the actions and column 3 briefs either the calculation or the conditions behind the actions.

\begin{tabular}{|c|c|c|}
\hline Step 1 & \multicolumn{2}{|c|}{$\begin{array}{l}\text { Initialize max_iteration, } N_{s r} \text { initial population } \\
\text { (Raindrops), } d_{\max } \text {, Pareto optimal Archive }\end{array}$} \\
\hline Step 2 & Initialize Sea & Best Raindrop by Eq. 6 \\
\hline Step 3 & Initialize Rivers & $\begin{array}{c}\text { Next }\left(\mathrm{N}_{\mathrm{sr}}-1\right) \text { best } \\
\text { Raindrops } \\
\end{array}$ \\
\hline Step 4 & Streams & Remaining Raindrops \\
\hline Step 5 & $\begin{array}{c}\text { Determine } \\
\text { [cost_raindrops] }\end{array}$ & By Eq. 6 \\
\hline Step 6 & Intensity of Flow & By Eq. 9 \\
\hline
\end{tabular}

Table 1 Flow of WCA Algorithm

\begin{tabular}{|c|c|c|}
\hline Step 7 & $\begin{array}{l}\text { Streams Flow to } \\
\text { Rivers }\end{array}$ & By Eq. 11 \\
\hline Step 8 & Rivers Flow to Sea & By Eq. 12 \\
\hline Step 9 & Swap Sea \& River & If $\operatorname{Cost}($ River $)<\operatorname{Cost}\left({ }_{\text {sea }}\right)$ \\
\hline Step 10 & Swap River \&Stream & If $\operatorname{Cost}\left({ }_{\text {Stream })}<\operatorname{Cost}_{(\text {River })}\right.$ \\
\hline Step 11 & $\begin{array}{l}\text { Evaporation \& } \\
\text { Precipitation of New } \\
\text { RainDrops }\end{array}$ & $\begin{array}{l}\text { If Evaporation condition } \\
\text { is satisfied }\end{array}$ \\
\hline Step 12 & Reduce $\mathrm{d}_{\max }$ & By Eq. 13 \\
\hline Step 13 & $\begin{array}{l}\text { Update the Pareto } \\
\text { optimal Archive } \\
\text { Removing the } \\
\text { dominated solutions }\end{array}$ & $\begin{array}{c}\text { Check whether Sea } \\
\text { dominates existing } \\
\text { Solutions in the Archive }\end{array}$ \\
\hline Step 14 & Go to Step 7 & $\begin{array}{l}\text { If \#_iteration } \\
<\text { max_iteration }\end{array}$ \\
\hline
\end{tabular}

\section{SIMULATION AND ANALYSIS}

The following scenario as shown in Table 2 was setup for comparing and analyzing the results. A moving target with a path similar to animals was used. The Mean residual Energy, Mean Standard Deviation of Residual Energy, Time of Death of Nodes and Prediction Accuracy were all studied for NSG and compared with IGA-BACA [9]. IGA- BACA is chosen, as it uses a metaheuristic approach for node scheduling.

Table 2 Simulation Settings

\begin{tabular}{|c|c|}
\hline Sensor Nodes & 100 \\
\hline Sensing Field & $1000 \mathrm{~m} \mathrm{X} \mathrm{1000} \mathrm{m}$ \\
\hline Transmission Range & $100 \mathrm{~m}$ \\
\hline Receiving Range & $100 \mathrm{~m}$ \\
\hline Initial Energy & $0.2 \mathrm{~J}-2 \mathrm{~J}$ \\
\hline Transmit Power & $35.28 \mathrm{e}-3 \mathrm{~W}$ \\
\hline Receiving Power & $31.32 \mathrm{e}-3 \mathrm{~W}$ \\
\hline Simulation Time & $20000 \mathrm{~s}$ \\
\hline
\end{tabular}

\subsection{Comparison of Residual Energy}

Table 3 Mean Standard Deviation of Remaining Energy

\begin{tabular}{|c|c|c|}
\hline \multirow{2}{*}{$\begin{array}{c}\text { Round (R) } \\
\text { 1R=100s }\end{array}$} & Mean Std. Deviation / Energy Mean \\
\cline { 2 - 3 } & NSG & IGA-BACA \\
\hline 10 & $0.122 / 0.295$ & $0.105 / 0.315$ \\
\hline 30 & $0.108 / 0.288$ & $0.101 / 0.291$ \\
\hline 60 & $0.067 / 0.280$ & $0.101 / 0.261$ \\
\hline 90 & $0.061 / 0.276$ & $0.100 / 0.247$ \\
\hline 120 & $0.059 / 0.271$ & $0.099 / 0.233$ \\
\hline 150 & $0.058 / 0.266$ & $0.099 / 0.219$ \\
\hline 180 & $0.056 / 0.261$ & $0.099 / 0.203$ \\
\hline 200 & $0.055 / 0.260$ & $0.098 / 0.200$ \\
\hline
\end{tabular}

Table 3 shows comparison of Mean Standard Deviation of Residual Energy and Mean Residual Energy of nodes across rounds. The performance of NSG is compared with IGABACA. The rate of decrease of residual energy in IGABACA is $36.5 \%$ whereas in NSG it is as low as $3.5 \%$.

Bayesian prediction predicts the occurrence of event in a region. This has greatly reduced the number of idle listening nodes waiting for the arrival of target or occurrence of event. Idle listening consumes as much power as needed by the 
receiving circuitry. This has greatly reduced rate of decrease of energy as time goes by, in NSG when compared to [9].

Mean Standard Deviation of Residual Energy gives a measure of the residual energy difference among the sensor nodes at a given point in time. When NSG's Std. Dev of Residual Energy is compared with that of IGA-BACA, it can be seen that the energy difference among the sensor nodes has been reduced greatly. This energy difference arises in IGA-BACA because of the onetime determination of the Sensor Cover based on the initial residual energy. In NSG the sensor covers are highly dynamic, as they are determined each time based on their residual energy. This decrease in energy difference also leads to a higher network lifetime.

\subsection{Comparison of Network Lifetime}

Figure 4 plots the Lifetime of the WSN. The decrease in the differences in residual energy among the sensor nodes plays the vital role in increasing the Lifetime of WSN when compared to IGA-BACA. The improved performance of NSG with time is explained by the increase in accuracy of Conditional Probability. The sensor nodes start the NSG when the learning of the target is still somewhat immature. But as time increases, the conditional probability gets more accurate which results in decrease in the number of Idle Listening Nodes and hence the increased Lifetime.

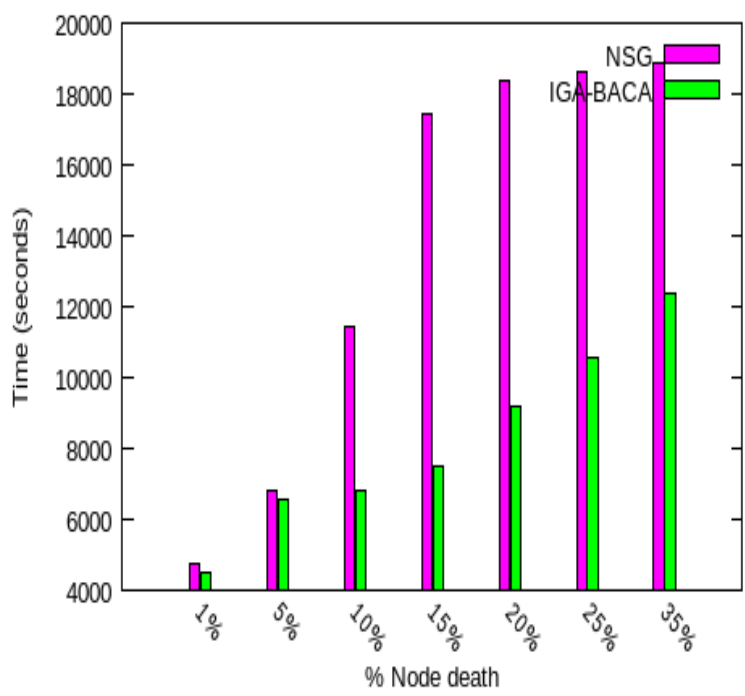

Fig 4: Lifetime of WSN

\subsection{Comparison of Prediction Accuracy}

Figure 5 plots the Prediction accuracy of Baye's Theorem. Prediction accuracy is measured as the ratio of accurate predictions to the actual occurrence of events in the sensing region. Initial rounds show higher miss in prediction by NSG. As time goes by, the accuracy increases. This is because as the target's trajectory is better understood, Conditional Probability gets more fine-tuned.

This results in NSG's prediction accuracy to be on par with IGA-BACA a fixed coverage set approach after 140 rounds. This also explains the improved performance by NSG in terms of Network Lifetime (Figure 4) as time increases.

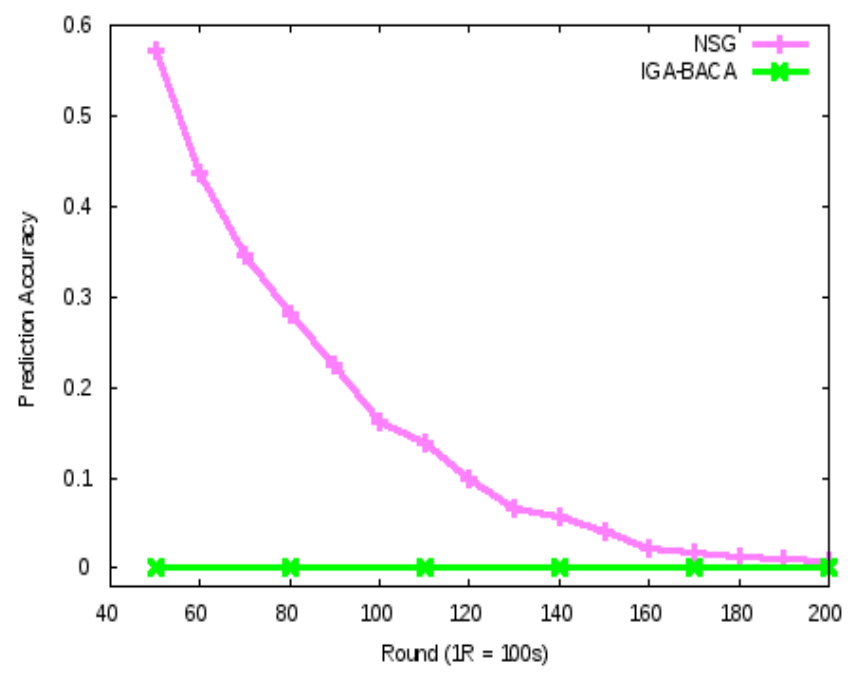

Fig 5: Comparison of Prediction Accuracy

\section{CONCLUSION}

A Node Scheduling approach based on Bayesian Probability and Game Theory has been proposed. The Node Scheduling Game is specifically proposed for moving targets or phenomenon that can be learnt and predicted. The sensor nodes study the trajectory of the moving target and using Bayesian Estimation predicts the future locality of occurrence of event. The sensor nodes in the predicted locality start a game with the goal of determining the appropriate number of sensor nodes that must stay awake. Using Water Cycle Algorithm, a Pareto optimal archive of solutions is determined and one solution among the archive is chosen as the sensor cover. Prediction of events combined with proportional expenditure of energy by sensor nodes has resulted in significant increase in Lifetime. Network Lifetime (35\% Node Death Time) is enhanced by $52 \%$ when compared to IGABACA. The sensor covers are chosen only based on the residual energy, but in future this can be extended to selection of node covers based on additional parameters like total coverage angle, total coverage area and WCA can be used for solving the multi-dimensional optimization problem.

\section{REFERENCES}

[1] Akyildiz, I.F., Su, W., Sankarasubramaniam, Y., and Cayirci, E., (2002). A survey on sensor networks.IEEE Communications Magazine, vol. 40, no. 8, pp. 102 -114 .

[2] Anastasi, G., Conti, M., Francesco, M., Passarella A. (2009). Energy Conservation in wireless sensor networks: A survey. Ad Hoc Networks, vol. 7, no.3, pp. 537-568.

[3] Leu, J., Chiang, T., Yu, M., and Su, K. (2015).Energy efficient clustering scheme for prolonging the lifetime of wireless sensor network with isolated nodes. IEEE communication letters, vol.19, no. 2, pp. 259-262.

[4] Mini, R., and Loureiro, A. (2012). Energyefficient design of wireless sensor networks based on finite energy budget. Computer Communications, vol. 35, no. 14, pp. 1736-1748.

[5] Rault, T., Bouadallah, A., and Challal, Y. (2014). Energy efficiency in wireless sensor networks: A top down survey. Computer Networks, vol. 67, pp. 104-122. 
[6] Torres, C., Glosekotter, P. (2011). Reliable and energy optimized WSN design for a train application. Journal of System Architecture, vol. 57, no. 10, pp. 896-904.

[7] Xiong, N., Huang, X., Cheng, H., and Wan, Z. (2013). Energy efficient algorithm for broadcasting in ad hoc wireless sensor networks. Sensors, vol. 13, no. 4, pp. 4922-4946.

[8] Manju, Satish, C., Bijender, K. (2016). Maximising network lifetime for target coverage problem in wireless sensor networks, IET Wireless Sensor Systems.vol. 6, no. 6, pp. 192-197.

[9] Jingwen, T., Meijuan, G., Guangshuang, G. (2016). Wireless sensor network node optimal coverage based on improved genetic algorithm and binary ant colony algorithm.EURASIP Journal on Wireless Communications, no. 1, pp. 104-114.
[10] Bo, j., Binoy, R., Hyeonjoong, C. (2013). ProbabilityBased prediction and Sleep Scheduling for Energy Efficient Target Tracking in Sensor Networks.IEEE Transactions on Mobile Computing, vol. 12, no. 4, pp. 735-747.

[11] Jakob, V.U. (2010). A Foray into the Worlds of Animals and Humans. (University of Minnesota Press, Minneapolis, London.

[12] Andrew, A.D., Kevin, C., Kazushige, O., Paul A.J. (2015). Cascading elastic perturbation in Japan due to the $2012 \mathrm{M}_{\mathrm{w}} 8.6$ Indian Ocean earthquake. Science Advances, vol. 1, no. 9 .

[13] Eskandar, H., Sadollah, A., Bahreininejad, A., andHamdi, M. (2012). Water cycle algorithm-A novel metaheuristic optimization method for solving constrained engineering optimization problems. Computers \& Structures, vol. 110,pp. 151-166. 